\title{
The European programme BORIS: Involvement of biological components in the transfer of radioactive nuclides to plants
}

\author{
C. Tamponnet, A. Martin-Garin, M.A. Gonze, A. Sanchez', N. Parekh', R. Vallejo ${ }^{2}$, T. Sauras ${ }^{2}$, \\ J. Casadesus ${ }^{2}$, C. Plassard ${ }^{3}$, S. Staunton ${ }^{3}$, L. Moberg ${ }^{4}$, R. Avila ${ }^{4}$, G. Shaw ${ }^{5}$ and C. Wells ${ }^{5}$ \\ Institute of Profection and Nuclear Safety, DPRE/SERLAB, \\ Cadarache, BP. 1, 13108 Saint-Paul-lez-Durance cedex, France \\ ${ }^{1}$ Center for Ecology and Hydrology, Merlewood Research Station, Cumbria, U.K. \\ ${ }^{2}$ Department of Plant Biology. University of Barcelona, Spain \\ ${ }^{3}$ INRA, Soil Science Unit, Montpellier, France \\ ${ }^{4}$ Swedish Radiation Protection Insfitute, Stockholm, Sweden \\ ${ }^{5}$ Imperial Callege, Berkshire, U.K.
}

\begin{abstract}
The ability to predict the consequences of an accidental release of radioactive nuclides relies mainly on the level of understanding of the mechanisms involved in radioactive nuclides interactions with different components of agricultural and natural ecosystems and their formalisation into predictive models. Numerous studies and databases about contaminated agricultural and natural areas have been obtained but their use to enhance our prediction ability has been largely limited by their unresolved variability. Such variability seems to stem from an incomplete knowledge about radioactive nuclide interactions with the soil matrix, soil moisture, biological elements in the soil and additional pollutants, which may be found in such soils.

In this project, we investigate mainly the role of the biological elements (plants, mycorrhiza, microbes) in: radioactive nuclide sorption/desorption in soils and radioactive nuclide uptake/release by plants. Because of the importance of the chemical nature of the involved radioactive nuclides, we will follow the bioavailability of three radioactive nuclides: caesium, strontium, and technetium. The role of additional non-radioactive pollutants will also be scrutinised as they may interfere with the mechanisms goveming radioactive nuclide transfer to plants. Knowledge acquired from the experiments will be incorporated into two mechanistic models BIOPHAST and BIORUR specifically modelling radioactive nuclide sorption/desorption from soil matrices and radioactive nuclide uptake by/release from plants. These mechanistic models will be incorporated into assessment models to enhance their prediction ability. Onc expects to extract from these experiments scientific bases for the development of bioremediation methods of radioactive nuclidecontarninated soils.
\end{abstract}

\section{INTRODUCTION}

Large environmental areas can be contaminated by the deposition of radioactive nuclides after a major nuclear accident. Moreover, uranium mining and milling, phosphate processing or coal mining produce various radioactive nuclides $\left({ }^{226} \mathrm{Ra},{ }^{238} \mathrm{U},{ }^{210} \mathrm{~Pb},{ }^{232} \mathrm{Th}\right.$ ) or pollutant trace elements (As, $\mathrm{Pb}, \mathrm{Cu}, \mathrm{Zn}, \ldots$ ) which may then be accumulated in surface soils of the surounding environment. In particular, contamination of cultivated and natural areas, which provide food, contributes to a great extent to population radiation dose. Crisis management and decision making largely depend upon our ability to predict how and to what extent such contamination may affect foodstuffs. This prediction ability relies mainly on the level of understanding of the mechanisms involved in radioactive nuclide interactions with different components of agricultural and natural ecosystems and their formalisation into predictive models.

Numerous studies and databases about contaminated agricultural and natural areas have been obtained from previous contamination events including nuclear accidents. The use of these previous data to enhance our prediction ability has been largely limited by their unresolved variability. Such variability may originate from incomplete knowledge radioactive nuclide interactions with the soil matrix, soil moisture, biological elements in the soil and additional pollutants, which may be found in such soils.

\section{OBJECTIVES OF THE BORIS PROGRAMME}

This scientific programme aims to fill gaps in our knowledge about mechanisms involved in radioactive nuclide contamination of terrestrial ecosystems by focusing on radioactive nuclide interactions with soil to improve :

- our understanding of the mechanisms governing their transfer to plants,

- their modelling for a better prediction,

- the development of bioremediation methods. 
The influence of key parameters and sources of variability, will be investigated throughout the entire project including :

- the physico-chemical nature of the radioactive nuclides (speciation),

- presence or not of additional non-radioactive pollutants,

either affecting the radioactive nuclide sorption/desorption with soil :

- the physico-chemical nature of the mineral, and organic components of soils,

- the biological components of soils,

- the soil organisation (intemal organisation of the different components of soils)

or the plant root uptake:

- physiological and biochemical variations of/in plants

- presence or not of associated mycorrhizal fungi.

PLANTS

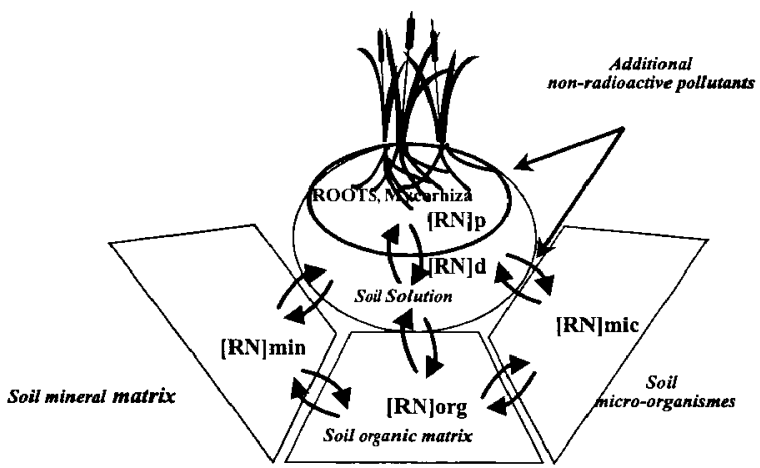

Figure 1: Schematic diagram showing the different radioactive nuclide pathways in the soil-plant system.

The first objective of this project is to improve our understanding of the mechanisms governing the transfer of radioactive nuclides to plants. To do so, we will mainly endeavour to comprehend the role of the biological elements involved in this transfer and/or found to be present in the structures and functions of the concerned radioactive nuclides.

In particular, the research aims at a better understanding of the factors governing radioactive nuclide sorption/desorption in soils to provide a better quantification of some key parameters such as soil moisture, soil solution $\mathrm{pH}$ and $\mathrm{pE}$, soil physics (partition in different solid phases), soil chemistry (chemical nature of the minerals involved in the solid phase of the soil) and biochemistry (chemical nature of the organic and biological phases of the soil).

The project also focuses on a better understanding of the factors controlling radioactive nuclide uptake/release by plants and the specific role of the plant rhizosphere, including the role of ectomycorrhizal fungi, and on the exact role of plants themselves (plant physiology, plant biochemistry). In these two research domains, we will address the exact role of additional pollutants on the bioavailability of radioactive nuclides.

Because of the importance of the chemical nature of the radioactive nuclides, we investigate in this project the bioavailability of three radioactive nuclides with different chemical properties:

- caesium, whose prevalent chemical form in the aqueous solution is the monovalent cation, $\mathrm{Cs}^{+}$

- strontium, whose prevalent chemical form in the aqueous solution is the divalent cation, $\mathrm{Sr}^{2+}$ 
- technetium, whose prevalent chemical form in oxic aqueous solution is a monovalent anion, $\mathrm{TcO}_{4}$ -

The second objective is to improve existing predictive models of radioactive nuclide interaction with soils by incorporating the knowledge acquired from the experimental results. This will be a three-step procedure:

- Specifying a conceptual and compartment model based initially on existing knowledge and on previously developed models.

- Developing mechanistic models of the processes occurring between the soil solid phase (mineral, organic, microbial) and the soil solution, and of the processes occurring between the plant system (including eventual mycorrhiza) and the soil solution, and validating these mechanistic models with the experimental results

- Integrating the results obtained into an improved compartment model, hopefully with reduced uncertainties of predictions compared to those of previous models.

The third objective is to extract from the experimental results some scientific basis for the development of bioremediation methods of radioactive nuclide-contaminated soils and to comprehend the role of additional non-radioactive pollutants on radioactive nuclide bioavailability.

These project objectives are fulfilled by integrating the development of models of radioactive nuclide transfer to plants with the experimental data obtained from laboratory experiments specifically designed to understand the role of some basic biological parameters and by describing the potential bioremediation methods based on the better understanding of the rote of biological elements in the bioavailability of radioactive nuclides in soils.

\section{BORIS WORK PLAN}

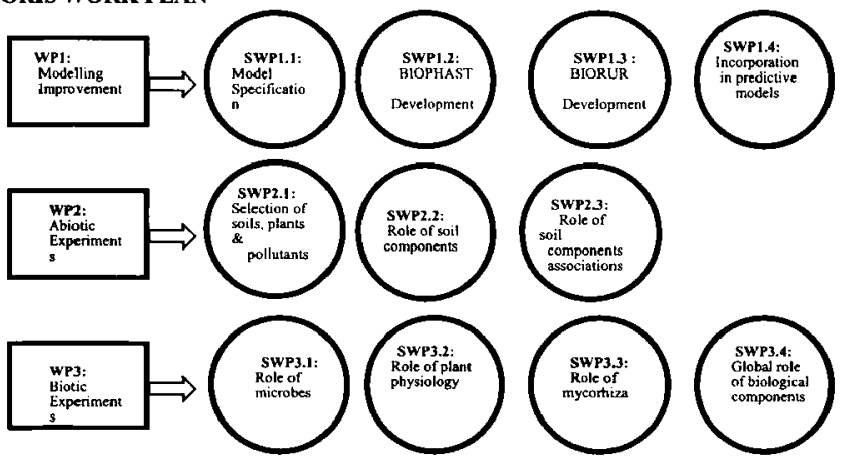

Figure 2: Schematic diagram showing the BORIS work plan

\subsection{WP1 Work Package 1: Improving predictive models}

The usual approach for evaluating radioactive nuclide uptake by plants from soil is by using concentration ratios, $C R$, which is defined as the ratio of radioactive nuclide concentration in plants $(\mathrm{Bq} / \mathrm{kg}$ dry or wet weight) to that in soil at a specified depth $(\mathrm{Bq} / \mathrm{kg}$ dry or wet weight). This is the approach recommended by the IAEA to use in models of radioactive nuclide uptake by agricultural plants. A review shows that the radiocaesium CR for agricultural plants can vary by a factor of up to 25 even for plants grown on the same site. Different types of soils lead to a variation by a factor of 50 . A variation from two to three orders of magnitude of the CR from soil to forest plants has been reported. Such high variability puts constraints upon the applicability of CR for assessments of radioactive nuclide transfer from soil to plants. In this project we will explore a possible solution to this problem by describing the bioavailability of radioactive nuclides in soils in mechanistic terms $[1,2]$. 
Table 1: Description of work for work package one.

SWP1.1 Specification of the conceptual and mechanistic models.

SWP1.2 Development and validation of soil/soil solution partition of radioactive nuclides BIOPHAST biogeochemical model.

- Model preparation,

- Model development,

- Model calibration and validation.

SWP1.3 Development and vatidation of soil solution/plant transfer of radioactive nuclides BIORUR mechanistic model.

- Model preparation,

- Model development,

- Model calibration and validation

SWP1.4 Integration of these mechanistic models into assessment models.

\subsection{Work Package 2: Abiotic processes affecting radioactive nuclides partition between Soil and Soll Solution}

In the soil system, the total pool of radioactive nuclides is distributed between the various solid components (with varying radioactive nuclide-binding capacities), and soil solution. A wide range of soil properties (e.g., $\mathrm{pH}, \mathrm{pE}$, organic matter and clay contents) and processes (such as microbial activity) are known to affect the solid-solution exchange and hence, soil solution composition. It is generally assumed that the free ion is the chemical species taken up by plants from soil solution during root uptake, and is thus the 'bioavailable' fraction. Changes in soil physico-chemical properties and biological processes are likely to affect the amount of radioactive nuclide in soil solution that is available for root uptake.

Table 2: Description of work for work package two.

SWP 2.1 : Sclection of soil types, soil components, associated plants and non-radioactive pollutants;

SWP 2.2: Specific role of selected soil components (minerals, otganics);

Exp.I. Sorption/desorption experiments of radioactive nuclides between main soil component and soil solution;

Exp.2. Effect of $\mathrm{pH}$, redox potential and water activity on the sorption/desorption of radioactive nuclides between main soil component and soil solution;

Exp.3. Effect of change in the chemical composition of the soil solution on the sorption/desorption of radioactive nuclides between main soil component and soil solution.

Exp.4. Effect of additional non-radioactive poltutant on the sorption/desorption of the radioactive nuclides between main soil component and soil solution.

SWP 2.3 : Role of associated soil components and soil organisation;

Exp.I. Sorption/desorption experiments of radioactive nuclides between soil/associated soil component and soil solution;

Exp.2. Effect of $\mathrm{pH}$, redox potential and water content on the sorption/desorption of radioactive nuclides between soil/associated soil component and soil solution;

Exp.3. Effect of change in the chemical composition of the soil solution on the sorption/desorption of radioactive nuclides between soil/associated soil componcnt and soil solution.

Exp.4. Effect of additional non-radioactive pollutant on the sorption/desorption of the radioactive nuclides between soil/associated soil component and soil solution.

The traditional approach used to assess the sorption capacity of soil solids for radioactive nuclides has utilised simple systems under controlled conditions to measure radioactive nuclide uptake and release, usually expressed in terms of the distribution coefficient, $K_{d}$ (the soil to soil solution concentration ratio). Such "uptake" (and "release") experiments typically employ the "batch technique', whereby soil (or contaminated soil) is equilibrated in suspension with a radiotracer in a background electrolyte (or a radioactive nuclide-free background electrolyte) at defined soil to solution ratios. The radioactive nuclides are measured in the solid and liquid phases following phase separation (using centrifugation, 
filtration, or dialysis bags) to derive $K_{\mathrm{d}}$. Interactions between soil organic matter and radioactive nuclides have also been studied experimentally using the $K_{d}$ approach, with soil extracts of organic components (e.g., humic and fulvic acids). The soluble components of soil organic matter (e.g., various low molecular weight products of plant and microbial metabolism) have also been studied as potential complexing agents and their effects on sorption processes investigated.

\subsection{WP3 Work Package 3 : Role of biological components on the transfer of radioactive nuclides}

\subsubsection{Potential role of micro-organisms}

The role of microbiological processes in the bioavailability and cycling of radioactive nuclides needs to be more fully elucidated in order to obtain parameters that are important for modelling and predicting the fate and transfer of radioactive nuclides in soils and soil-plant systems. To date, few studies have characterised the full potential of soil micro-organisms to cycle radioactive nuclides in soils [3].

Many studies have measured the uptake of radioactive nuclides by organic soils but the role of soil microorganisms may have been masked by the presence of even small amounts of clay minerals occurring in these soils. Our main objective in this work package (SWP3.1) is to provide mechanistic in formation on various aspects of soil-microbe-radioactive nuclide interactions, which will be, used in the geochemical and root uptake modeks being considered in WP1. We intend to set up a series of experiments using a selected forest soil (see SWP2.1), a selected agricultural soil (see SWP2.1) and a biologically active, 'mineral-free', organic soil produced in the laboratory from a mixture of plant litters, to determine the potential of soil micro-organisms to accumulate different radioactive nuclides in situ. Biological uptake and release will be differentiated from abiotic processes by comparing experimental results to those determined in SWP2.2 and 2.3 and by using irradiated (sterilised) soils and pure and mixed cultures of specific groups of soil micro-organisms.

These results highlight the need to develop an innovative experimental approach, which incorporates microbial activity at optimal conditions of growth, in order to address the role of soil micro-organisms on radioactive nuclide sorption/desorption.

The role of different groups of soil micro-organisms on radioactive nuclide uptake will be determined in a clay-free soil and also in a mineral soil system.

\subsubsection{Role of plant}

Soil to plant transfer of radioactive nuclides is not determined only by the concentration of radioactive nuclides in the soil solution [4]. Thus, the use of empirically obtained transfer factors should be replaced by a more mechanistic approach.

It has been established empirically that the root uptake of $\mathrm{Cs}^{+}, \mathrm{Sr}^{2+}$ and $\mathrm{TcO}_{4}-$ are largely affected by the $\mathrm{K}^{+}, \mathrm{Ca}^{2+}$ and $\mathrm{NO}_{3}$ concentration in the root medium respectively [5].

Also, some seasonal or developmental variation in soil to plant transfer have been reported showing that a constant coefficient for relating plant accumulation of radioactive nuclide with soil solution composition may not be appropriate.

The idea of root uptake of $\mathrm{Cs}^{+}, \mathrm{Sr}^{2+}$ and $\mathrm{TcO}_{4}^{+}$through the same pathways as $\mathrm{K}^{+}, \mathrm{Ca}^{2+}$ and $\mathrm{NO}_{3}^{-}$ respectively has long been proposed. One hypothesis is that $\mathrm{Cs}^{+}, \mathrm{Sr}^{2+}$ and $\mathrm{TcO}_{4}$ and respectively $\mathrm{K}^{+}, \mathrm{Ca}^{2+}$ and $\mathrm{NO}_{3}$ are competing for the same transporters. Another explanation could be the following: the plant could adjust the kinetics of the uptake system in response to the balance between $\mathrm{K}^{+}, \mathrm{Ca}^{2+}$ and $\mathrm{NO}_{3}^{-}$ supply and demand for growth. The plant does indeed regulate both the activity of transporters and their rate of synthesis in order to adjust the uptake to the requirements for growth.

They could vary also as a consequence of environmental conditions, agronomic practices or presence of xenobiotics in a way that can be predicted by most crop models. Thus, the effect on radioactive nuclide transfer, of diverse factors affecting crop development can be simulated with radioecological model using as input data the output of any external crop model.

\subsubsection{Potential role of mycorrhizal fungi}

The aim of this investigation is to determine how ectomycorrhizal infection modifies the uptake and translocation of radioactive nuclides [6]. The uptake of radioactive nuclides by mycorrhizal and non mycorrhizal tree seedlings from contaminated soils will be measured. Furthermore, the specially designed rhizosphere culture device will enhance chemical changes in the (myco-)rhizosphere and allow them to be quantified and their consequences on the availability of radioactive nuclides to be assessed. A two-compartment rhizosphere device may also be adapted, separating mycorrhizal roots and fungal hyphae, to determine separately uptake by these organs. 
The additional effect of non radioactive metal pollution on radioactive nuclide uptake will also be investigated. A screening of the fungi in pure culture will allow a pollutant metal and a sensitive fungus to be selected for detailed studies in association with tree seedlings. It should thus be possible to distinguish between possible effects on fungal growth and survival, capacity for mycorrhizal infection and the uptake and translocation properties.

Table 3: Description of work for work package three

SWP 3.I Role of soil micro-organisms in radioactive nuclides bioavailability in soil.

Exp. I: Studying the role of microbial community composition in the potential uptake/desorption of selected radioactive nuclides, using large-scale microcosm experiments.

Exp. 2: Radioactive nuclide sorption/desorption by a mineral-free organic soil and impact of addition of selected minerals. Exp.3: Studying the role of major groups of micro-organisms on radioactive nuclide sorption/desorption in selected soils.

Exp. 4: Measuring the effects of some physical and chemical factors on the biological sorption/desorption of radioactive nuclides.

SWP 3.2 Role of variations in plant physiology and biochemistry on radioactive nuclides transfer to plants.

Exp.1 : Predicting soil solution radiocacsium concentration at the root surface.

Exp.2 : Physiological mechanisms detcrmining the uptake of radioactive nuclides.

Exp.3 : Effects of additional pollutants on plant root uptake of radioactive nuclides.

SWP 3.3: Role of mycorrhiza in radioactive tuclides transfer to plants

Exp.1. Studying the effect of the presence or absence of ectomycorrhizal fungi associated with tree species on the chosen radioactive nuclides transfer to plant (selected tree species).

Exp.2. Studying the effect of additional non-radioactive pollutant on the mycorrhizal activity on the chosen radioactive nuclides bioavailability. Measuring the potential growth inhibition of the fungi by non-radioactive pollutant in pure culture. Comparing the effect of additional non-radioactive pollutant on transfer of radioactive nuclides on treated (mycorhizal infection) and non-treated tree.

SWP 3.4: Global role of biological components on radioactive nuclides transfer to plants.

Soil column experiments with:

'sterile' or non-sterile agricultural soil, either planted or not planted with the selected plant, either inoculated or not with a carefully selected additional non-radioactive pollutant.

'sterile' or non-sterile forest soil, either planted or not planted with the selected mycorrhiza-infected or non-infected plant, either inoculated or not with a carefully selected additional non-radioactive pollutant.

\section{References}

[1] Avila R. and Moberg L., J. Environ. Radioact. 45 (1999) 271-282.

[2] Avila R., M.A. Gonze, C. Tamponnet, J. Casadesus, G. Shaw. BORIS Technical Note 1: Model Specifications.2001.

[3] Tamponnet C., Plassard C., Parekh N., Sanchez A., "Impact of micro-organisms on the fate of radioactive nuclides in rhizospheric soils", in International Congress on radioecology and ecotoxicology of terrestrial and estuarine environments ECORAD 2001, September 3-7, 2001, Aix-en-Provence, accepted.

[4] Shaw G. and Bell J.N.B., J. Environ. Radioact. 13 (1991) 283-296.

[5] Sauras T., Vallejo V., Valcke E., Colle C., Förstel H., Millàn R. Jouglet H., J. Environ. Radioact. 45 (1999) 191-217.

[6] Entry J.A., Astrud L.S. and Reeves M., Environ. Pollution 104 (1999) 449-457. 